\title{
Preparation and Application of the Hydrophilic Amino-Silicone Softener by Emulsion Polymerization
}

\author{
Ruza Artikboeva*, Mingxing Yang*, Yue Wu, Chen Jie, Quan Heng\# \\ Chemistry Department, Wuhan Textile University, Wuhan, China \\ Email: ruza1994artikboyeva@gmail.com,3354723331@.qq.com, "quanheng2002@163.com
}

How to cite this paper: Artikboeva, R., Yang, M.X., Wu, Y., Jie, C. and Heng, Q. (2020) Preparation and Application of the Hydrophilic Amino-Silicone Softener by Emulsion Polymerization. Advances in Chemical Engineering and Science, 10, 1-23.

https://doi.org/10.4236/aces.2020.101001

Received: October 20, 2019

Accepted: November 2, 2019

Published: November 5, 2019

Copyright $\odot 2020$ by author(s) and Scientific Research Publishing Inc. This work is licensed under the Creative Commons Attribution International License (CC BY 4.0).

http://creativecommons.org/licenses/by/4.0/

(c) (i) Open Access

\begin{abstract}
The amino poly-siloxane was modified with self-made polyether silicones by the crafts of emulsion polymerization. We studied the emulsifier, the dosage of emulsifier initiator, polyether/silane coupling agent, $\mathrm{pH}$ value. There are some results of the research indicated in the following. First, there are many factors for the hydrophilic amino-silicone softener both on the polymerization process and the performance, such as the proportion and amount of emulsifier, initiator dosage, the mass ratio of the polyether and hydrogen silicone oil, the choice of silicone coupling agent, and the ratio and dosage of polyether silicones silane coupling agent. Second, the amount of hydrogen of the hydrogen silicones and the choice of catalyst are the key points on the synthesis of polyether silicones. The amount of hydrogen should be low and the catalyst must be economical and efficient and its introduction should be small and times, other more the holding time is not too long. The next one is that, in the process of hydrolysis of silane coupling agent, we need some acid to adjust $\mathrm{pH}$ value, or they will be broken down. The most important one but not the last is that the epoxy group can improve the products low-temperature supple and the persistence of the finishing fabric, furthermore it can reduce yellowing and has a good stability of the inorganic salt, however, it will be destroyed if the reaction temperature for epoxy is too high. What's more, it is very economical and environmental that the process of emulsion is simple and with less emulsifier.
\end{abstract}

\section{Keywords}

Amino-Silicone Softener, Polyether, Emulsifier, Hydrophilic, Softness and Smoothness, Emulsion Polymerization

${ }^{*}$ Co-first author. 


\section{Introduction}

In dyeing and finishing applications, in order to make the fabric have a smooth, soft handle and improve the quality of finished products, besides the use of rubber blanket mechanical treatment to adjust the handle (improve the displacement performance of the interlacing point of the fabric), softeners are often used to finish the fabric. In the post-finishing of natural fibers, various resins are used, which can prevent shrinkage, wrinkle, fast drying and ironing, and make the fabrics have some superior properties of synthetic fibers, but the handle will also become rough and hard. Therefore, softeners must be added to the resin working fluid or in the post-treatment bath to finish the natural fibers. Cotton-type or medium-length chemical fiber blended fabrics, such as: tampon/cotton, tampon/viscose, polyester/nitrile, etc., all require soft, smooth and other style characteristics. In order to solve these problems better, soft finishing has become one of the indispensable processes. In addition, woolen, silk and knitted fabrics also need to be softened for wear ability. Especially in recent years, with the continuous development and application of superfine fibers, the corresponding super softeners are needed to meet the needs of the market. Therefore, soft finishing has become an important post-finishing process in textile printing and dyeing process, which is essential for the quality of textile products and the added value of products.

Softeners are textile auxiliaries which can reduce the friction coefficient between fibers to obtain the surface softness and lubrication effect of fibers. Softeners for textiles must have the following characteristics [1] [2] [3]:

1) Good softness.

2) It has good compatibility with other additives.

3) It has no allergic or stimulating effect on human body and no harmful gas emerges during processing.

4) There is no yellowing or discoloration, which does not affect the fastness of dyes to sunlight.

5) Good washing resistance.

6) It meets the requirements of high temperature treatment in dyeing and finishing.

7) The price is moderate.

8) The finishing process is simple.

9) The storage performance is stable, biodegradable and meets the requirements of environmental protection.

Silicone has many outstanding advantages such as lubricity, softness, hydrophobicity, film-forming, etc. In addition, this kind of material is non-toxic, non-environmental pollution, low cost, and has been widely used in various industries. Since the 1970s, with the development of organosilicon chemistry, organosilicon softener as a new type of molecule softener has developed rapidly. Compared with traditional non-silicon softeners, silicone softeners exhibit better softening properties because of their lower rotational free energy than $\mathrm{C}-\mathrm{O}$ 
bonds. Therefore, silicone softener has been rapidly adopted by textile enterprises in a few decades since its appearance, and gradually replaced the traditional non-silicone softener to become the dominant softener variety [4]. Silicone softener has become a kind of softener with wide application, good performance and outstanding effect in textile industry.

\section{The Purpose, Significance and Main Contents of This Study}

Silicone softeners are widely used because of their excellent performance and stability. In recent years, the most studied softeners are modified silicone compounds, such as polyether/amino silicone oil softeners. Compared with the early modified organo-siloxane, they have more functional groups and more complex structure. The treated fabrics have good handle and hydrophilicity, good tensile, tear resistance and durable pressing properties. They do not cause fading and yellowing of the fabrics, and can improve the washing fastness and sweat resistance. In the preparation of hydrophilic amino-silicone softener, hand feeling and hydrophilicity are always contradictory. Good hand feeling, poor hydrophilicity or good hydrophilicity, but the decline of hand feeling is a common problem of this kind of softener, and it is difficult to achieve the unity of hand feeling and hydrophilicity. In addition, the yellowing problem of amino-silicone softener needs further solution [5] [6] [7]. In order to solve these problems, people use the excellent hydrophilicity of polyether group and high activity of epoxy to blend amino silicone oil. The obtained silicone oil can be emulsified with water only with a small amount of emulsifier, and the stability of the emulsion is good. The treated fabric has excellent handle and hydrophilicity. At the same time, the reaction of epoxy group with amino group plays the role of shielding and protecting amino group. It not only maximizes the excellent softness of amino group, but also prevents the fabrics from yellowing easily after baking at high temperature. It also gives the fabrics good antistatic and stains resistance, improves the tear strength of the fabrics, and enhances the added value of the products.

Polyether silicone oil was modified by cationic emulsion polymerization instead of the traditional cationic hydroxyl silicone oil emulsion. The hydrophilicity of amino silicone oil softener was solved. The effects of $\mathrm{pH}$ value of pre-emulsion, dosage of self-made polyether silicone oil, reaction temperature and holding time on the properties of softener were discussed.

\section{Preparation and Modification Principle of Amino Silicone Oil}

\subsection{Selection of Basic Polymeric Monomers}

The properties of hydrophilic amino silicone oil softener depend on the properties of polymer monomers, the reactivity of monomers, the amount of grafting and the arrangement of monomers in the molecular chain. In actual selection, attention should be paid not only to the reactivity of monomers, i.e. the binding 
force with fibers or other monomers, but also to the viscosity, appearance, stability of diluted emulsions and film formation on fibers of the synthesized silicone oil, as well as to various factors such as cost and environmental protection. The effects of various monomers on the properties of hydrophilic amino silicone softeners are shown in Table 1.

Table 1 shows that the introduction of silane coupling agent or diethylamine and ethylenediamine containing amino group can improve the finishing effect of silicone oil softener. However, yellowing occurs in varying degrees, and the finished fabric is not hydrophilic. Therefore, polyether containing hydrophilic group and epoxy containing reactive group should be introduced. Active polyether1\# and active polyether2\# and F-6 all contain hydrophilic groups. Active poly-ethers have good smoothness and antifouling properties. F-6 has high reaction activity and is convenient for grafting. Silicone oil emulsion is synthesized.

The solution has good hydrophilicity and poor washing ability. Epoxy has high reactivity and can be attached with various hydrophilic groups at its end to improve its hydrophilicity. The crosslinking reaction between epoxy modified silicone oil and amino alkyl groups in amino silicone oil molecules can effectively

Table 1. The properties of monomers.

\begin{tabular}{|c|c|}
\hline Monomer & Performance characteristics \\
\hline D4 & The content of silicon is high, up to $37.6 \%$. It is easy to open. \\
\hline $\begin{array}{l}\text { Hydrogen-containing } \\
\text { silicone oil active } \\
\text { polyether }\end{array}$ & $\begin{array}{l}\text { Under certain conditions, the } \mathrm{Si}-\mathrm{H} \text { bond is easy to react with amines and dilute hydrocarbons to prepare various } \\
\text { modified silicone oils. It gives the fabric good hydrophilicity, antistatic property, smoothness and antifouling property; } \\
\text { synthetic silicone oil is water-soluble and can be emulsified without additional emulsifier; however, the softness of } \\
\text { finished fabric is poor. }\end{array}$ \\
\hline & Characteristics of iso-active polyether 1. \\
\hline Active polyether2\# & $\begin{array}{l}\text { It contains double bonds and hydrophilic groups. It has high reactivity and strong hydrophilicity. The water washing } \\
\text { resistance of the synthesized softener is poor. }\end{array}$ \\
\hline F-6 & $\begin{array}{l}\text { Under certain conditions, the Si-H bond is easy to react with amines and dilute hydrocarbons to prepare various } \\
\text { modified silicone oils. It gives the fabric good hydrophilicity, antistatic property, smoothness and antifouling property; } \\
\text { synthetic silicone oil is water-soluble and can be emulsified without additional emulsifier; however, the softness of } \\
\text { finished fabric is poor. }\end{array}$ \\
\hline EpoxyAE-6 & $\begin{array}{l}\text { It contains reactive groups and reacts with the groups of } \mathrm{OH}, \mathrm{NH}_{2} \text { and } \mathrm{COOH} \text { on the surface of the fibers to improve } \\
\text { the durability, softness, waterproof and durable finishing effect of the finished fabric. It is not yellowing and has good } \\
\text { stability to inorganic salts. The end of the fabric can be connected with hydrophilic groups. }\end{array}$ \\
\hline Silane-coupled mustard & It contains strong polar amino groups, which can interact with light and carboxyl groups on the fibers. \\
\hline KH-550 & $\begin{array}{l}\text { So that the friction coefficient between the fibers decreases, thus achieving a good finishing effect, but the finished } \\
\text { fabrics tend to yellowish. }\end{array}$ \\
\hline Silane coupling agent & The diamine coupling agent has neat molecular structure, good orientation and adsorb-ability. \\
\hline $\mathrm{KH}-602$ & But its diamine structure is easier to oxidize, which makes the light-colored fabric yellowing seriously after finishing. \\
\hline Ethylenediamine & $\begin{array}{l}\text { The simplest diamine has strong alkalinity and reactivity. The finished fabric with amino silicone oil synthesized by } \\
\text { diamine has good handle, but serious yellowing and easy cross-linking. }\end{array}$ \\
\hline Two ethylamine & $\begin{array}{l}\text { Amino silicone oil has low boiling point, mild reaction conditions and good handle, but its alkalinity is too weak. A } \\
\text { small amount of alkali catalyst is needed to increase the reaction rate. }\end{array}$ \\
\hline
\end{tabular}


block amino groups, inhibit yellowing or discoloration of softeners, improve the stability of working fluid, do not damage the style of amino silicone oil, and give it better softness and smoothness.

Therefore, considering the properties and practical operability of the polymer monomers, the stability and production cost of the silicone oil softener emulsion synthesized, the silane coupling agents $\mathrm{KH}-550$ and $\mathrm{KH}-602$, as well as diethyl-amine, were selected as the introduction objects of amino functional groups; active poly-ethers and F-6 were used simultaneously to optimize the optimum proportion formula; the viscosity and hydrogen content of hydrogen-containing silicone oil, etc. The development of hydrophilic amino silicone oil softener was emphasized. The third substance needed to prepare emulsion with certain stability is called emulsifier. Most of the emulsifiers are surfactants. The function of emulsifier is that when it disperses on the surface of dispersed substance, it forms a film or double electric layer, which can make the dispersed phase charged, thus preventing the small droplets of dispersed phase from coagulating with each other and making the formed emulsion more stable.

\subsection{Selection and Classification of Emulsifiers}

Although emulsifiers do not directly participate in chemical reactions in the polymerization system, they are one of the most important components. The type and concentration of emulsifiers will directly affect the initiation rate and chain growth rate. It also affects the molecular weight and molecular weight distribution of polymers that determine the properties of polymers, as well as the concentration of latex particles related to the properties of emulsions, the size and size distribution of latex particles, etc. The selection of emulsifier is not only related to the stability of emulsion system, the normal production process, and the safety and reliability of storage and application, but also to the cost of polymer.

In the emulsification method, the most important condition for preparing relatively stable emulsion is the choice of emulsifier after the properties of oil and water phases are fixed. Due to the variability of oil and water phases and the different types of emulsions required, it is virtually impossible to find a general good emulsifier. In other words, only after specifying the composition and properties of oil and water phases and the type of emulsion required, the appropriate method is adopted.

Good surface activity and the ability to reduce surface tension require that emulsifiers can be adsorbed at the interface, rather than completely dissolved in any phase.

The emulsifying properties of emulsifiers are related to their affinity with oil or water phases. Oil-soluble emulsifier is easy to get W/O emulsion, water-soluble emulsifier is easy to get $\mathrm{O} / \mathrm{W}$ emulsion. Mixed use of two emulsifiers with higher oil solubility and water solubility sometimes has better emulsifying effect. Correspondingly, the greater the polarity of oil phase, the greater the hydrophilicity of emulsifier is required; the smaller the polarity of oil phase, the stronger the hydrophobicity of emulsifier is required. 
There are two commonly used methods to select emulsifiers: HLB method (hydrophilic-lipophilic equilibrium method) and PIT method (phase transition temperature method). The former is suitable for various types of surfactants, while the latter is a supplement to the former method and only suitable for non-ionic surfactants.

The HLB value is defined as a number of surfactants. The larger the number, the stronger the hydrophilicity, and the smaller the number, the stronger the hydrophobicity. The HLB value is $\mathrm{O} / \mathrm{W}$ emulsifier in the range of $8-18$, while the HLB value needed for silicone oil to emulsify into $\mathrm{O} / \mathrm{W}$ emulsion is about 10.5.

The commonly used emulsifiers can be divided into the following three categories:

1) Cationic emulsifier. Cationic emulsifiers can dissociate surface active ions with positive charges in water. Most of these emulsifiers are suitable for preparing W/0 emulsions. Most of its molecules contain nitrogen atoms, which are derivatives of organic amines, including various ammonium salts and quaternary ammonium salts.

For example, dodecyl, (dimethyl) benzyl ammonium bromide, $\mathrm{C}_{12} \mathrm{H}_{25}$ $(\mathrm{Me})_{2} \mathrm{PhCH}_{2} \mathrm{~N}^{+} \mathrm{Br}^{-}$, (DBDA), its hydrophilic quaternary ammonium terminal group is the active cation.

2) Anionic emulsifier. The common characteristics of anionic emulsifiers are high emulsifying efficiency, effective reduction of surface tension, low dosage, small micelles and formed emulsion particles. However, because of its ionic properties, the emulsion particles are charged, which makes them sensitive to the electrolyte freeze-thaw process and other emulsifier varieties to varying degrees, resulting in reduced stability, easy foaming and difficult to eliminate. Such as dodecyl-benzenesulfonic acid, $\mathrm{C}_{12} \mathrm{H}_{25} \mathrm{C}_{6} \mathrm{H}_{4} \mathrm{SO}_{3}^{-} \mathrm{H}^{+}$(DBSA) its hydrophilic sulfonic acid end groups are living anions.

3) Non-ionic emulsifier. Non-ionic emulsifiers are insensitive to the acidity and alkalinity of the medium and the presence of high valent metal ions. It is easy to adjust the hydrophilic and lipophilic groups in the molecule when synthesizing.

The size of the hydrophilic group in particular facilitates the control of the type of emulsion used to form it. For example, dodecyl poly-oxyethylene ether, $\mathrm{C}_{12} \mathrm{H}_{25}\left(\mathrm{OC}_{2} \mathrm{H}_{4}\right)_{6} \mathrm{OH}$, which takes poly-oxyethylene chain as its hydrophilic segment, acid or base catalyst can be converted from its emulsifier. For example, quaternary ammonium salts are usually used with hydroxides of alkali metals, such as $\mathrm{MOH}\left(\mathrm{M}=\mathrm{K}, \mathrm{Na}, \mathrm{Li}, \mathrm{NH}_{4}\right)$. In fact, quaternary ammonium bases act as catalysts.

$\mathrm{C}_{12} \mathrm{H}_{25}(\mathrm{Me})_{2}\left(\mathrm{C}_{6} \mathrm{H}_{5} \mathrm{CH}_{2}\right) \mathrm{N}^{+} \mathrm{Br}^{-}+\mathrm{MOH}-\mathrm{C}_{12} \mathrm{H}_{25}(\mathrm{Me})_{2}\left(\mathrm{C}_{6} \mathrm{H}_{5} \mathrm{CH}_{2}\right) \mathrm{N}^{+} \mathrm{OH}^{-}+\mathrm{MBr}$,

$\mathrm{C}_{12} \mathrm{H}_{25} \mathrm{C}_{6} \mathrm{H}_{5} \mathrm{SO}_{3}$ itself is an acid, which can emulsify and catalyze. However, non-ionic emulsifiers can stabilize the emulsion and cannot play a catalytic role.

Therefore, at present, cationic and non-ionic, anionic and non-ionic emulsifiers are commonly used in emulsion polymerization. The products obtained have the characteristics of fine particles, low foam and stability. 


\subsection{Amino Silicone Oil Softener and Its Emulsion Polymerization Principle}

Silicone polymers are characterized by soft texture, low surface energy and physiological inertia [8]. Used in textile finishing can improve handle, waterproof and anti-static, etc. The organosilicon molecule used in these places is most economical, convenient and safe in the form of emulsion. Amino poly-siloxane emulsion has different softening and smoothing effects on cellulose fibers, protein fibers, synthetic fibers and their blends. Cotton, viscose fibers and polyester/cotton fabrics can get soft hand feeling without bones after treatment. If water emulsion is prepared from silicone oil emulsification, it can only be made of small molecular weight. Silicone oil with high molecular weight is difficult to emulsify because of its high viscosity and poor stability, so it is usually prepared from ring-opening emulsion polymerization [9]. This process has been very popular in China in recent years and has been applied in many aspects.

\section{Characteristics of Emulsion Polymerization}

Emulsion polymerization is the polymerization of monomer and water in the prepared emulsion under the action of emulsifier. The system consists of four basic components: monomer, water, emulsifier and initiator dissolved in water. Compared with other methods of polymerization, such as bulk polymerization, solution polymerization and suspension polymerization, emulsion polymerization has the following important characteristics:

1) The continuous phase of the emulsion polymerization system is water. The polymerization reaction takes place in the latex particles dispersed in the water phase. Although the viscosity of the latex particles is very high, the viscosity of the whole reaction system is not high, basically close to that of the continuous phase water, and the viscosity of the system will not change greatly during the polymerization process. Therefore, the emulsion polymerization system is easy to heat dissipation and will not appear local. Heat leads to the failure of synthesis, and explosion is not easy to occur.

2) In the emulsion polymerization system, the initiator dissolves in the water phase, and decomposes into free radicals continuously in the water phase. The individual free radical chains are separated in the isolated latex particles. The electrostatic repulsion force exists due to the charged surface of latex particles, which makes the latex particles suspended stably in the water phase and cannot collide with each other. In other words, the free radical chains in different latex particles cannot terminate each other, but can only terminate with the primary free radicals diffused by water phase. Therefore, the chain termination rate of emulsion polymerization is lower than that of bulk polymerization, and the average life of free radicals in emulsion polymerization is longer. If a polymer molecule chain has enough time to grow, it can grow into a large polymer, so the relative molecular weight of emulsion polymerization is higher than that of bulk polymerization. On the other hand, under the premise of constant reaction temperature and initiator concentration, low termination rate will lead to higher 
free radical concentration, so the free radical concentration in emulsion polymerization system is higher than that in bulk polymerization system, so the reaction rate in emulsion polymerization is higher than that in bulk polymerization system [10] [11] [12].

3) Medium water is non-flammable, non-explosive, non-toxic, tasteless, not polluting the environment, safe in production and harmless to human body. It can greatly improve the working conditions of polymerization workshop, post-treatment workshop and other application processes. In addition, water is cheap and easy to obtain, which can significantly reduce the cost and avoid the trouble of solvent recovery by solution polymerization.

4) Compared with other methods of polymerization, emulsion polymerization has the advantages of simple equipment and process, convenient operation and great flexibility. It can be produced by batch method, semi-continuous method or continuous method, high elasticity of production and small yield, and emulsion polymerization can be used to prepare polymers whether dissolved in water, slightly dissolved in water or insoluble in water. Homo-polymerization of a single monomer can also be carried out by copolymerization of two or more monomers. Graft polymers, directional polymers, interpenetrating network polymers, core-shell and heterogeneous polymers can also be prepared with various specificities [13] [14].

In many cases, the polymer emulsion made is a product itself, which is directly used as paint, adhesives or other working substances in the construction, textile, paper-making, industrial painting, leather and other industries.

Emulsion polymerization, like bulk polymerization, requires acid or alkali catalysis, but it must be assisted by emulsifiers. Emulsifier can disperse insoluble poly-siloxane into tiny particles in water, and polymerize on its surface. After polymerization, the synthesized polymer can be surrounded, making it difficult to coagulate and become a stable emulsion.

\subsection{Principle of Emulsion Polymerization}

Emulsion polymerization is a method of producing polymer by free radical addition polymerization in water or other liquid emulsion, in which isolated latex particles are formed according to micelle mechanism or oligomer nucleation mechanism.

According to the time-conversion relationship, the discussion of the mechanism of emulsion polymerization can be divided into four stages: dispersion stage, stage I (formation stage of latex particles), stage II (growth stage of latex particles) and stage III (completion stage of polymerization reaction).

\subsubsection{Dispersion Phase}

At this stage, emulsifiers are added in batches. The initially added emulsifier is dissolved in water in the form of a single molecule, which is the true solution. When the concentration of emulsifier reaches CMC, the emulsifier begins to appear in the form of micelles. When the monomer is added into the system, it is 
dispersed into droplets under stirring force. Some emulsifiers are adsorbed on the surface of the droplets to form a monolayer. The hydrophilic end of the emulsifier molecule points to the water phase, while the lipophilic end points to the center of the droplets, so that it can be suspended stably in the water phase. In addition, due to the solubilization of micelles, some monomers dissolved in water will be attracted to micelles from aqueous phase to form so-called solubilized micelles.

\subsubsection{Phase I (Latex Particle Formation Phase)}

When water-soluble initiator was added into the system, the initiator began to decompose into free radicals in the aqueous phase at a certain reaction temperature. Before the polymerization reaction, there is often a non-reaction induction period during which the free radicals generated are intercepted by oxygen or other inhibitors in the system without polymerization. After the induction period, the reaction acceleration period, namely Stage I, is entered. In stage I, the free radicals of initiator decomposition diffuse into a solubilized micelle, in which polymerization is initiated and macromolecular chains are formed, thus the micelle becomes a polymer emulsion colloidal particle swelled by monomers, i.e. latex particles. Because the formation of latex particles mainly occurs in this stage, it is also called the formation stage of latex particles.

Polymerization occurs in latex particles. As the reaction proceeds, the monomers in latex particles are gradually consumed. The monomers in the water phase in the form of free molecules continue to diffuse into the latex particles to supplement, while the dissolved monomers in the water phase come from the monomer droplets. Thus, the monomers establish a dynamic equilibrium among the latex particles, the water phase and the monomer droplets. Since the monomer is consumed by the polymerization reaction in the latex particles, the equilibrium moves along the direction of the monomer droplets, water phases and latex particles.

In Stage I, emulsifiers have four applications, namely micelle emulsifier, emulsifier dissolved in water in the form of single molecule, emulsifier adsorbed on the surface of monomer droplets and emulsifier adsorbed on the surface of latex particles, which can also establish a dynamic balance between them.

As the nucleation process proceeds, more and more new latex particles will be formed; at the same time, the surface area of latex particles will gradually increase as the size of latex particles continues to grow. As a result, more and more emulsifiers are transferred from aqueous phase to the surface of latex particles, so that the quantity of emulsifiers dissolved in aqueous phase decreases continuously, so that the balance between the emulsifiers dissolved in aqueous phase and the micelles that have not yet nucleated is maintained. It is destroyed and the equilibrium moves towards the direction of micelle-water phase-latex particles. As a result, the amount of micelle emulsifier gradually decreases, some micelles are destroyed, and the nucleation process itself also consumes micelles, resulting in fewer and fewer micelles and eventually disappear. 


\subsubsection{Phase II (Latex Particle Growth Phase)}

In stage II, the micelles disappeared, while the emulsifiers would be distributed in three locations, i.e., dissolved in water, adsorbed on the surface of latex particles and adsorbed on the surface of monomer beads. The emulsifiers in these three positions are also in dynamic equilibrium.

In Phase II, monomers, emulsifiers and free radicals form a certain balance among latex particles, monomer droplets and water phase. In the reaction zone, monomers are constantly consumed, and monomer balance moves along the direction of monomer droplets, water phase and latex particles, resulting in the monomer droplets decreasing gradually until the monomer droplets disappear.

\subsubsection{Phase III (Completion of Polymerization)}

In Phase III, micelles and droplets were not present, only latex particles and aqueous phase. The distribution of emulsifiers, monomers and free radicals is determined by the dynamic equilibrium between the two phases. Because the monomer droplets do not exist, the polymerization reaction in the latex particles can only consume the monomers stored by themselves, but cannot be supplemented, so the concentration of polymer in the latex particles will increase, the internal viscosity will increase, and the macromolecules will become entangled with each other, resulting in the activity of free radical chains reduced, and the resistance of two free radicals to terminate when they spread together will increase. With the increase of conversion, the chain termination rate constant decreased sharply.

\subsection{Emulsion Polymerization}

\subsubsection{Cationic Emulsion Polymerization}

Cationic organosilicon emulsion polymerization has the following requirements [7] [8].

1) Poly-siloxane must contain $\mathrm{Si}-\mathrm{H}$ chains, which can be heated to dehydrogenate the hydroxyl groups on the surface of the fabric to form chemical bonds to make the adhesion firm; 2) Emulsifiers can be heated to decompose, and do not remain on the surface of the fabric, so cationic emulsifiers containing quaternary amines, such as dodecyl, (dimethyl) benzyl ammonium bromide, whose hydrophilic quaternary ammonium terminal groups are required. It is an active cation. $\mathrm{Si}-\mathrm{H}$ component is added to the emulsion, and the emulsifier is decomposed by heat treatment above $150^{\circ} \mathrm{C}$ to strengthen adhesion. The emulsion synthesized in this way has good handle, but hydrophobic effect, poor hydrophilicity of the fabric, and subsequent improvement is difficult. In addition, cationic emulsion polymerization is often accompanied by bleaching oil, resulting in low solid content [15].

Data show that $\mathrm{D}_{4}$ can be ring-opening polymerized with $\mathrm{NH}_{4} \mathrm{OH}$ or $\mathrm{NaOH}$ as catalyst and DBDA as emulsifier at $50^{\circ} \mathrm{C}-90^{\circ} \mathrm{C}$. After equilibrium, $\mathrm{D} 4$ can be neutralized by glacial acetic acid and ether is stratified. Conversion rate is measured by drying method and molecular weight of polymer is measured by viscos- 
ity method. The results show that the reaction between $50^{\circ} \mathrm{C}-90^{\circ} \mathrm{C}$ can reach equilibrium. After equilibrium, the conversion of the ring is generally about $87 \%$, which is generally consistent with bulk polymerization. Researchers believe that the dosage of emulsifier is $2.7 \%$, which is 10 times of the critical micelle concentration (CMC). $\mathrm{D}_{4}$ enters the micelle from the aqueous phase, polymerizes in the micelle and produces polymer chains.

\subsubsection{Anionic Emulsion Polymerization}

The condensation of the intermediate in anionic emulsion polymerization on the surface of the emulsion particles results in the growth of silica chains. Anionic emulsion polymerization is easy to operate and has no bleaching problem. It has been widely used in textile finishing. Its polymerization process is similar to cationic emulsion polymerization [16]. However, because of the repulsion between anions and cellulose, it is difficult to adsorb and form films on the surface of cellulose. Therefore, the fabric treated by anionic emulsion polymerization has good hydrophilicity, but poor handle.

Weyenberg first used DBSA $\left(\mathrm{C}_{12} \mathrm{H}_{25} \mathrm{C}_{6} \mathrm{H}_{4} \mathrm{SO}_{3} \mathrm{H}\right)$ and its sodium salt emulsification polymerization $\mathrm{D}_{4}$ to open up the way of emulsion polymerization. The conversion rate was determined by drying method and the molecular weight was measured by viscosity method. It was concluded that the condensation of $\mathrm{HOD}_{\mathrm{n}} \mathrm{H}$ intermediates on the surface of emulsion particles resulted in the growth of silica chain. Musenchang studied the reaction process. He used GLC to analyze the conversion rate and GPC to analyze the molecular weight and its distribution. There were two peaks in the GPC spectrum. With the increase of conversion, the peak of small molecular weight gradually decreased while the peak of large molecular weight gradually increased, reaching 60,000. When the temperature is low, the growth and decline of the two peaks are obvious at $40^{\circ} \mathrm{C}$ and still exist at $60^{\circ} \mathrm{C}$ and $80^{\circ} \mathrm{C}$, but not as obvious as at $40^{\circ} \mathrm{C}$. Increasing the amount of sodium salt in emulsifier DBSA also delayed the appearance of macromolecular peaks. These results are similar to cationic emulsion polymerization and can be explained by the viewpoint of cationic emulsion polymerization.

\section{Material and Program}

\subsection{Instruments and Reagents}

In Table 2, we have given raw materials, reagents, instruments and equipment.

Table 2. Raw materials and reagents.

\begin{tabular}{cccc}
\hline Name of raw material & Abbreviation & Specifications & Manufacturer \\
\hline Octamethyl-cyclotetrasiloxane & D4 & Industrial grade & Jiangxi Starfire Silicone Plant \\
Low-hydro silicone oil & & Industrial grade & Jiangxi Jiayi New \\
Hydrogen-containing ring & & Materials Co., Ltd. \\
& Industrial grade & Guangzhou Huamei \\
& & & Chemical Co., Ltd. \\
\hline
\end{tabular}




\section{Continued}

\begin{tabular}{|c|c|c|c|}
\hline Epoxy & AE-6 & \multicolumn{2}{|l|}{ Industrial grade } \\
\hline Active polyether $1 \#$ & & \multicolumn{2}{|l|}{ Industrial grade } \\
\hline Active polyether $2 \#$ & & \multicolumn{2}{|l|}{ Industrial grade } \\
\hline Allyl alcohol poly-avoidance & F-6 & \multicolumn{2}{|c|}{$\begin{array}{c}\text { Industrial grade } \begin{array}{c}\text { Zhejiang Real Madrid Chemical } \\
\text { Industry Group Co., Ltd. }\end{array}\end{array}$} \\
\hline Silane coupling agent & & Industrial grade & $\begin{array}{l}\text { Nanjing Yude Heng Fine } \\
\text { Chemical Co., Ltd. }\end{array}$ \\
\hline $\begin{array}{l}\text { Dodecyl trimethyl } \\
\text { ammonium chloride }\end{array}$ & 1231 & Industrial grade & $\begin{array}{l}\text { Shanghai Jinshan Jingwei } \\
\text { Chemical Co., Ltd. }\end{array}$ \\
\hline $\begin{array}{l}\text { Octadecyl trimethyl } \\
\text { ammonium chloride }\end{array}$ & 1831 & Industrial grade & $\begin{array}{l}\text { Shanghai Jinshan Jingwei } \\
\text { Chemical Co., Ltd. }\end{array}$ \\
\hline Polyethylene glycol 400 & PEG & $\mathrm{AR}$ & $\begin{array}{l}\text { Jiangsu Pisheng } \\
\text { Chemical Co., Ltd. }\end{array}$ \\
\hline AEO Series & AEO & Industrial grade & Zhejiang Transfar Co., Ltd. \\
\hline Dodecylbenzene sulfonic acid & SDBA & Industrial grade & $\begin{array}{l}\text { Guangdong Jiangmen } \\
\text { Xinke Chemical Co., Ltd. }\end{array}$ \\
\hline XL Series & $\mathrm{XL}$ & Industrial grade & BASF Chemical Industry \\
\hline Ethylenediamine & & $\mathrm{AR}$ & $\begin{array}{c}\text { Tianjin Damao } \\
\text { Chemical Reagent Factory }\end{array}$ \\
\hline Diethylamine & & $\mathrm{AR}$ & $\begin{array}{c}\text { Yixing brilliant } \\
\text { Chemical Reagent Factory }\end{array}$ \\
\hline Isopropanol & & $\mathrm{AR}$ & $\begin{array}{l}\text { Yixing brilliant Chemical } \\
\text { Reagent Factory }\end{array}$ \\
\hline Glacial acetic acid & HAc & $\mathrm{AR}$ & $\begin{array}{c}\text { Yixing Yamao } \\
\text { Chemical Raw Materials Factory }\end{array}$ \\
\hline Potassium hydroxide & & $\mathrm{AR}$ & $\begin{array}{l}\text { Yixing Yamao Chemical } \\
\text { Raw Materials Factory }\end{array}$ \\
\hline Catalyzer & & $1 \%$ & Self-control \\
\hline \multicolumn{4}{|c|}{ Instruments and Equipment } \\
\hline Instrument & Model & & Manufactor \\
\hline Electronic balance & BS6(8)A & Yuyao Jinn & uo Balance Instrument Co., Ltd. \\
\hline One thousandth electronic balance & BS124S & Instr & $\begin{array}{l}\text { Sedoris Scientific } \\
\text { uments (Beijing) Co., Ltd }\end{array}$ \\
\hline Electronic valuation scale & ACS-6 & Mete & $\begin{array}{l}\text { Shanghai Pudong } \\
\text { ering Instrument Factory }\end{array}$ \\
\hline $\begin{array}{l}\text { Digital display constant } \\
\text { temperature water and oil bath pot }\end{array}$ & R201D & Shang & hai Yuhua Ware Co., Ltd. \\
\hline $\begin{array}{c}\text { Temperature regulating electric } \\
\text { heating sleeve }\end{array}$ & $\mathrm{KDM}$ & Yong & $\begin{array}{l}\text { Shandong Juancheng } \\
\text { gxing Instrument Factory }\end{array}$ \\
\hline Electronic constant speed mixer & JHS-1 & Hangzho & u Instrument Motor Factory \\
\hline Intelligent Digital Whiteness Meter & WSB-3A & Wenzhou & Ludong Instrument Factory \\
\hline $\begin{array}{l}\text { Pad dyeing experiment machine } \\
\text { (small rolling car) }\end{array}$ & $\mathrm{P}-\mathrm{AO}$ & Jiangsu Jing & jiang Hailan Equipment Factory \\
\hline
\end{tabular}




\section{Continued}

\begin{tabular}{|c|c|c|}
\hline Rotary viscosimeter & NDJ-79 & $\begin{array}{l}\text { Shanghai Changji } \\
\text { Geological Instrument Co., Ltd. }\end{array}$ \\
\hline Electric centrifuge & $800 \mathrm{~B}$ & Jiangsu Jintan Xinhang Articles Factory \\
\hline Jiuyang soybean milk machine & JYDZ-16C & Guangzhou Dianmei Trading Co., Ltd. \\
\hline Automatic interfacial tensiometer & JYW-2(8)A & $\begin{array}{l}\text { Chengde Xinguo Testing } \\
\text { Equipment Company }\end{array}$ \\
\hline $\mathrm{PH}$ acidity meter & $\begin{array}{c}\text { Thunder magnet } \\
\text { PHS-3C }\end{array}$ & Shanghai Jingke Ware Co., Ltd. \\
\hline Amplitude heat setting machine & $\mathrm{R}-3$ & Taiwan RAPID Company \\
\hline Fabric tear tester & YG033A & $\begin{array}{l}\text { Nantong Hongda Experimental } \\
\text { Instruments Co., Ltd. }\end{array}$ \\
\hline Digital whiteness meter & DSBD-1 & Wenzhou Ludong Instrument Factory \\
\hline $\begin{array}{l}\text { Electrothermal constant } \\
\text { temperature blast dryer }\end{array}$ & DHG-9070A & SHANGHAI SOSPECT WARE CO., LTD. \\
\hline
\end{tabular}

\subsection{Experimental Method}

\subsubsection{Preparation of Hydrophilic Amino Silicone Oil Emulsion by Emulsion Polymerization}

1) Anionic emulsion polymerization

Pre-emulsion was prepared by adding non-ionic emulsifier and deionized water in $2000 \mathrm{ml}$ plastic cup in turn, then adding DMC slowly while stirring in the lower part of the mixer.

After the pre-emulsification, deionized water, anionic emulsifier, non-ionic emulsifier and silica coupling agent were put into three flasks with thermometer and stirring rod. The temperature was increased to $50^{\circ} \mathrm{C}$ by stirring, and the pre-emulsion was dripped in $90-120$ minutes, then the temperature was increased to $75^{\circ} \mathrm{C}-80^{\circ} \mathrm{C}$, and the heat preservation time was 6 hours. Anionic amino silicone oil emulsion is obtained by cooling.

2) Cationic emulsion polymerization

Pre-emulsion was prepared by fully hydrolyzing silane coupling agent with partial deionized water, fully dissolving cationic emulsifier with residual deionized water, fully mixing the two solutions and slowly adding them under rapid agitation of mixer.

D40 put the bottom part (emulsifier, PEG400, alkali catalyst and deionized water) into three flasks with thermometer and stirring rod. Slowly stir and heat up to $80^{\circ} \mathrm{C}$, hold for 10 minutes, then slowly add the pre-emulsifying part.

The cationic amino silicone oil emulsion was obtained by cooling and acidizing for 6 hours at $80^{\circ} \mathrm{C}$ and 2 hours at $70^{\circ} \mathrm{C}$ after dripping.

\subsubsection{Polyether Silicone Oil Was Modified by Cationic Emulsion Polymerization}

Hydrogen-containing silicone oil, active polyether and isopropanol were put into three flasks, heated to $100^{\circ} \mathrm{C}$ and added $1 / 3$.

When the exothermic temperature of the reaction becomes stable, the re- 
maining catalyst is added to the self-made catalyst. After the full reaction with hydrogen, the self-made polyether silicone oil is synthesized by holding $30 \mathrm{mi}-$ nutes at $105^{\circ} \mathrm{C}$.

1) Polyether silicone oil and DMC were hydrophilically modified by adding self-made polyether silicone oil in the pre-emulsion part. The dropping temperature of polyether silicone oil and DMC in the polymerization part was reduced to $75^{\circ} \mathrm{C}$, and the holding time was unchanged.

2) Polyether silicone oil was directly modified by direct modification of polyether silicone oil. The effects of different hydrogen content or different structure of silicone oil and different structure of polyether or polyether with different molecular weight on the synthesis of polyether silicone oil were investigated, and the synthesis process was optimized. Then the hydrophilic amino-silicone oil emulsion was prepared by cationic emulsion polymerization.

3) Two or more polyether silicone oils with different tendencies were synthesized by blending different polyether silicone oils. The polyether silicone oils were proportionally put into plastic cups, and the solution of fully dissolved emulsifier and coupling agent was added while stirring, and the remaining water was evenly stirred. In three flasks with thermometers and stirring bars, the measured water and alkali catalyst solution are added, and the prepared emulsion is stirred and added. The temperature is raised to $80^{\circ} \mathrm{C}$, and the change of the state of the emulsion is observed. The optimum synthesis process was obtained.

\subsection{Testing and Characterization Methods}

\section{Emulsion Stability Test z}

1) Centrifugal stability

$20 \%$ hydrophilic amino-silicone oil emulsion was placed in centrifugal tube and centrifuged for 10, 15, 20 and 30 minutes at $2000 \mathrm{r} / \mathrm{min}$. The stratification and crust were observed visually.

2) Mechanical stability

The emulsion diluted to a certain concentration was sheared at high speed for $0 / 3 / 5 / 10 / 15$ min on Jiuyang soybean milk machine and the particle size of the emulsion was detected by hand to see whether there were small particles in the emulsion or on the wall.

3) Thermal stability

In $250 \mathrm{ml}$ beaker, weigh $4 \mathrm{~g}$ hydrophilic amino silicone oil softener diluted in proportion, add $196 \mathrm{ml}$ of water, stir it evenly with glass rod, and then put it in a water bath at $50^{\circ} \mathrm{C}$ for 30 minutes at constant temperature to observe the change of solution state. If the temperature continues to rise, there will be no oil drift and turbidity in the solution at $60^{\circ} \mathrm{C}, 70^{\circ} \mathrm{C}$ and $80^{\circ} \mathrm{C}$, which indicates that the thermal stability of the emulsion is better, and vice versa.

4) Alkali resistance stability

On the basis of measuring the heat resistance stability, the $\mathrm{pH}$ value is adjusted to $10-10.5$ with $10 \%$ caustic soda solution, and then put into a water bath 
pot at $50^{\circ} \mathrm{C}$ for 30 minutes at constant temperature. The change of solution state is observed and the temperature continues to rise. If there is no bleaching oil and turbidity in the solution at $60^{\circ} \mathrm{C}, 70^{\circ} \mathrm{C}$ and $80^{\circ} \mathrm{C}$, it shows that the alkali resistance of the emulsion is better, on the contrary, it is worse.

5) Electrolyte resistance stability

On the basis of measuring the heat-resistant stability, add $1 \mathrm{~g}$ sodium sulfate and put it in a $50^{\circ} \mathrm{C}$ water bath pot for 30 minutes after full dissolution. Observe the change of solution state and continue to heat up. If there is no bleaching oil and turbidity in the solution at $60^{\circ} \mathrm{C}, 70^{\circ} \mathrm{C}$ and $80^{\circ} \mathrm{C}$, it shows that the electrolyte-resistant stability of the emulsion is better, and vice versa.

\subsection{Application Performance Testing}

\subsubsection{Softness}

The test was carried out with a self-made softness tester. The surface of the platform is smooth and smooth, the front incline of the platform is 41.5 self-angle with the bottom of the platform; the sample plate is $15 \mathrm{~cm}$ long and $5 \mathrm{~cm}$ wide, and can drive the sample to move synchronously; there is a ruler on the side of the platform to measure the length of the cloth strip.

The sample is $15 \mathrm{~cm}$ a $5 \mathrm{~cm}$ in longitude and latitude; the sample is placed on the top of the platform, pressed on the pressure plate, one end of the sample is aligned with the pressure plate, and coincides with the "zero" scale of the scale; then the pressure plate is pushed out at the speed of $0.3-0.5 \mathrm{~cm} / \mathrm{s}$, like the inclined edge of the cloth until the top of the sagging cloth just touches the inclined edge. Record the length of the sagging part of the cloth strip, and repeat the test with the reverse side of the cloth strip, and take its average value.

\subsubsection{Hydrophilicity}

A standard dropper ( $25 \mathrm{drops} / \mathrm{ml}$ ) is used to measure the time taken for the fabric to absorb a drop of water from the surface of the fabric spreading horizontally $2 \mathrm{~cm}$ above the fabric.

\subsubsection{Whiteness (High Temperature Yellowing Resistance)}

The spectral reflectance of finished fabrics was measured by DSBD-1 digital whiteness meter. Compared with the blank sample, the whiteness value of the finished fabric is larger, that is, the whiteness difference between the finished fabric and the blank fabric is smaller, and the color change of the fabric is smaller.

\subsubsection{Comprehensive Performance (Smoothness, Fluffy)}

5 - 10 form a group, touch and grade the finished fabric, and divide the test effect into five grades, with the unfinished blank cloth as one grade, the best is five grades.

\section{Results and Discussion}

\subsection{Synthesis of Polyether Silicone Oil}

The water solubility, emulsifying property and surface activity of polyether sili- 
cone oil depend on its molecular structure, including the composition and length of polycinnamoxil chain, the ratio, content and end-group properties of hydrophilic chain segment.

In the preparation of polyether silicone oil, the choice of hydrogenated silicone oil and catalyst is the key. The viscosity of hydrogenated silicone oil directly affects the efficiency of siloxane addition and the compatibility between hydrogen silicone oil and the ring (emulsion stability and application performance). The activity of the catalyst is an important factor to ensure the product's low color, low odor, stability, short reaction time, complete reaction and mild conditions. In addition, the structure, molecular weight and amount of active polyether also affect the ring opening and grafting amount. The viscosity of polyether cinnamon oil also has some influence on the handle and moisture absorption of fabric finishing. The ratio of cinnamon coupling agent to polyether is the key to coordinate the handle and hydrophilicity.

\subsection{Composition and Ratio of Emulsifier}

The functions of textile auxiliary industry to emulsifiers are varied and complex. So far, no single emulsifier has been found that can undertake a variety of functions, so it is necessary to compound the emulsifier. Moreover, emulsifier compounding can also reduce the amount of a single variety and improve efficiency, because different emulsifiers have different chemical structures or compositions, but when they are used at the same time, they generally have synergistic effect with each other.

In the experiment of cationic emulsion polymerization, we chose 1231 and 1831 compound to change the ratio of 1231/1831 to carry out experiments and test the properties of the synthetic softener products. The results are shown in Table 3.

Table 3 shows that changing the ratio of emulsifier 1231 to 1831 has little effect on tear strength of treated fabrics. However, when the ratio of emulsifier 1231 is low, the stability of the synthesized emulsion is not very ideal, and there will be different levels of delamination and turbidity. With the increase of the

Table 3. 1231/1831 influences on the properties of softener products.

\begin{tabular}{cccc}
\hline $1231 / 1831(\mathrm{w} / \mathrm{w})$ & $1.7 / 1$ & $2.0 / 1$ & $2.4 / 1$ \\
\hline Centrifugal stability & Layered & Good & Good \\
Thermal stability & Good & Good & Good \\
Alkali resistance stability & Turbid & Good & Good \\
Salt-tolerant stability & Turbid & Good & Good \\
Softness $(\mathrm{mm})$ & 25.6 & 22.3 & 24.7 \\
Hydrophilicity & 9.56 & 9.47 & 8.32 \\
Whiteness & 75.8 & 76.3 & 76.5 \\
Tear strength $(\mathrm{N})$ & 12.8 & 12.4 & 12.6 \\
\hline
\end{tabular}


1231 ratio, the stability of the synthesized emulsion will increase. When 1231/1831 reaches $2.0 / 1$, the stability of the synthesized emulsion is good, and there is no layering, oil bleaching and so on, and the softness of the treated fabric is also raised. It is not the yellowing degree that has been greatly improved (the whiteness value increases). This is because 1231 and 1831 are hydrophilic emulsifiers, but 1231 is more hydrophilic, while 1831 is hydrophilic with polyether silicone oil which is biased towards emulsifying and lipophilic. Therefore, increasing the 1231 ratio is beneficial to the improvement of the properties of the emulsion; however, if the proportion of 1231 continues to increase, the stability of the synthetic emulsion is not stable. The hydrophilicity and whiteness of treated fabrics are improved, but the softness of treated fabrics seems to be decreasing. Therefore, considering the comprehensive consideration, we choose the ratio of 1231 and 1831 to $2.0 / 1$, the emulsifying effect of this ratio is good, and the comprehensive performance of the synthetic emulsion treated fabric is the best.

\subsubsection{The Dosage of Emulsifier}

Maintaining emulsion stability is a necessary condition for emulsion polymerization. The stability of emulsion is affected by the kinds of emulsifiers, the concentration of emulsifiers, and various factors that affect emulsification of emulsifiers.

Various factors that affect emulsification of emulsifiers affect the stability of emulsions.

According to Table 4, when the amount of emulsifier is relatively small (10\%), the performance of the emulsion is not satisfactory in all aspects. This is because when the emulsifier concentration is low, only the surface of latex particles is covered by emulsifier molecules. Under such conditions, latex particles tend to coalesce, and large latex particles are produced by small latex particles, which will coagulate seriously, resulting in emulsion layering, reducing the quality of products and making use of them. When the amount of emulsifier is moderate, monomer can disperse and solubilization before polymerization, and form stability. The monomer emulsion provides a place for initiating polymerization in

Table 4. Effect of emulsifier dosage on product performance.

\begin{tabular}{cccc}
\hline $\begin{array}{c}\text { Emulsifier dosage (for oil phase) } \\
\text { Quality, \% }\end{array}$ & 10 & 15 & 20 \\
\hline $\begin{array}{c}\text { Centrifugal stability } \\
\text { Thermal stability }\end{array}$ & Layered & No stratification, no crust & Crust \\
Alkali resistance stability & Turbid & Good & Good \\
Salt-tolerant stability & Bleaching oil & Good & Good \\
Softness (mm) & 26.3 & Good & Muddy pond \\
Hydrophilicity & 10.27 & 26.4 & 27.1 \\
Whiteness & 76.9 & 9.58 & 10.54 \\
Tear strength $(\mathrm{N})$ & 13.6 & 13.8 & 76.3 \\
\hline
\end{tabular}


the polymerization process, monomer swelling gel, which is also adsorbed on the surface of latex particles, stabilizing latex particles, preventing condensation after polymerization and neutralization, ensuring that the emulsion has the appropriate viscosity, good stability and the best handle when processing the fabric; but when the emulsifier is excessive (20\%), the polymerization rate is too fast. The excessive amount of micelle in the emulsion has a certain effect on the stability of the product, and the addition of the emulsifier will inevitably result in the decrease of the effective components in the unit mass and the decrease of the product performance. Therefore, we must reasonably choose the dosage of emulsifier. In conclusion, the optimum dosage of emulsifier is $15 \%$ (for oil phase quality).

\subsubsection{Effect of Polyether Wafer Oil Dosage on Hydrophilic Amino Wafer Oil Softener}

Polyether silicone oil was synthesized from active polyether and hydrogen-containing silicone oil, and then copolymerized with octamethylcyclotetrasiloxane, polyethylene glycol and cinnamon coupling agent to form hydrophilic amino cinnamon oil softener. It is known that the structure of polyether (including molar weight, the ratio of hydrophilic group to hydrophobic group), the type of terminal group (alkoxy group, alkyl group and acyloxy group), the specification of polydimethylsiloxane (the ratio of $\mathrm{Me}_{2} \mathrm{SiO}$ chain to $\mathrm{MeHSiO}$ chain) and the ratio of polyether to polycinnamoxane have important effects on the water solubility and surface activity of copolymers. Therefore, the amount of polyether silicone oil (that is, the ratio of polysiloxane $\mathrm{D}_{4}$ ) is an important factor affecting the comprehensive properties of the synthesized hydrophilic amino silicone oil emulsion.

Table 5: Properties of hydrophilic amino cinnamon softeners synthesized from different dosages of polyether cinnamon oil (mass ratio to $\mathrm{D}_{4}$ ).

Note: 1) Only the ratio of polyether silicone oil to $\mathrm{D}_{4}$ was changed, and the ratio of emulsifier, silica coupling agent, initiator and water remained unchanged.

2) Centrifugal stability test: rotational speed $20 \mathrm{r} / \mathrm{min}$, time $30 \mathrm{~min}$.

It can be seen from Table 5 that the centrifugal stability of the polyether silicone oil and the emulsion synthesized by $\mathrm{D}_{4}$ is good, indicating that the grafted

Table 5. The influence of the amount of polyether silicone dosage on the product performance.

\begin{tabular}{cccccc}
\hline $\begin{array}{c}\text { Dosage of polyether } \\
\text { silicone oil (for D4 mass ratio) }\end{array}$ & $0.9 / 1$ & $1.2 / 1$ & $1.5 / 1$ & 1.6/1 & $1.7 / 1$ \\
\hline Centrifugal stability & $\begin{array}{c}\text { Not stratified, not } \\
\text { knotted }\end{array}$ & $\begin{array}{c}\text { Not stratified, not } \\
\text { knotted }\end{array}$ & $\begin{array}{c}\text { Not stratified, not } \\
\text { knotted }\end{array}$ & $\begin{array}{c}\text { Not stratified, not } \\
\text { knotted }\end{array}$ & $\begin{array}{c}\text { Not stratified, not } \\
\text { knotted }\end{array}$ \\
Skin & Skin & Skin & Skin & 25.3 & 28.6 \\
Softness (mm) & 24.5 & 25.1 & 25.5 & 6.32 & 4.58 \\
Hydrophilicity & 13.56 & 9.12 & 76.4 & 76.4 & 76.5 \\
Whiteness & 76.4 & 76.6 & 12.6 & 12.6 & 12.4 \\
Tear strength (N) & 12.4 & 12.8 & & & \\
\hline
\end{tabular}


ring and polyether are in good condition. With the increase of polyether proportion, the hydrophilicity of the synthesized product is strong, but the softness decreases. When the ratio is increased to a certain ratio (1.7/1), the hydrophilicity slightly improves, and the softness decreases obviously. Because polyether contains hydrophilic group instead of reactive group, the increase of polyether content in a certain range can greatly improve the hydrophilicity of the fabric, but it does not play a role in improving the softness of the fabric; whiteness and breaking strength are not affected by the change of proportion. Therefore, we choose the ratio of active polyether to $\mathrm{D}_{4}$ as 1.6/1.

\subsubsection{Selection of Coupling Agent}

There are many kinds of silane coupling agents. The general formula can be written as $\mathrm{RSiX}_{3}$. Among them, $\mathrm{R}$ is an active group with affinity and reaction ability with polymer molecules, such as vinyl, chloropropyl, epoxy and amino groups, and $\mathrm{X}$ is an enanthoxy group which can hydrolyze, such as methoxy, ethoxy, etc. Its coupling action is often simply described as the formation of covalent bond bridges between polymers and fillers by orderly molecular layers of cinnamon system. The coupling effect of cinnamon firing coupling agent on filling systems containing polar groups or introducing polar groups is obvious. The different $\mathrm{R}$ groups in the coupling agents make different kinds of copolymerization suitable for the coupling agents, because the $\mathrm{R}$ group is selective to the reaction of the polymers.

According to the properties of silane coupling agents, $\mathrm{KH}-550, \mathrm{KH}-171$ and $\mathrm{KH}-602$ and their mixtures were selected. But because they are very susceptible to environmental impact (they are easy to hydrolyze and have high $\mathrm{pH}$ requirements), it is difficult to mix them.

Table 6 examines the effects of coupling agent monomers on the properties of products synthesized by the same polymerization process.

Experiments show that the handle of silicone oil emulsion containing vinyl but without amino KH-171 is obviously better than that of KH-550 and KH-602 containing amino groups. This is because the amino-containing silane coupling agent can effectively bind epoxy, phenolic, melamine, polyurethane and other resins, and the fiber molecules contain hydroxyl, termination and other polar groups, so silane coupling agent containing amino group is generally selected as fabric softener [17]. There are two different chemical functional groups in the

Table 6. The influence of different silane coupling agent on the softener.

\begin{tabular}{cccc}
\hline Selected coupling agent & KH-550 & KH-171 & KH-602 \\
\hline Stability & Good & Good & Good \\
Softness (mm) & 29.3 & 30.6 & 28.9 \\
Smoothness & $4-5$ & $4-5$ & $4-5$ \\
Hydrophilicity & 11.39 & 12.47 & 12.52 \\
Whiteness & 74.9 & 75.2 & 73.6 \\
\hline
\end{tabular}


structure of amino cinnamon coupling agent, i.e. the hydroxyl group and the amino group. The hydroxyl group hydrolyzes to form silanol and then reacts with the silanol group of organic cinnamon. The strong polar amino group interacts with the light and carboxyl groups on the fiber to obtain good interfacial bonding. Moreover, KH-602 (N--(-amino ethyl)--propyl methyl two methoxy cinnamon) is a diamino coupling agent, while $\mathrm{KH}-550$ contains only one amino group. The silicone oil emulsion synthesized by KH-602 has better orientation and adsorption properties when processing fabrics, and the friction coefficient between the fibers is reduced to a minimum, so that the fabric has better softness. Therefore, KH-602 cinnamon coupling agent was selected in this experiment.

Table 7 shows the influence of coupling agent and polyether monomer proportions dosage on the softener.

Note: 1) Only the ratio of coupling agent to polyether silicone oil is changed. The ratio of emulsifier, $\mathrm{D}_{4}$, initiator and water are unchanged.

2) Centrifugal stability test: rotational speed $20 \mathrm{r} / \mathrm{min}$, time $30 \mathrm{~min}$.

Therefore, in the process of emulsion polymerization, the suitable amount of coupling agent is $\mathrm{w}=3.00 \%$ (the quality of polyether).

\subsubsection{Determination of Polymerization Process}

In emulsion polymerization, the introduction of coupling agent, the preparation of pre emulsion, the $\mathrm{pH}$ value of pre emulsion, the dripping acceleration, the temperature and holding time of emulsion polymerization will affect the stability and performance of polymer emulsion.

Because silane coupling agents are easy to produce flocs or hydrolysates when they encounter water molecules, we choose to use a certain amount of water to dissolve the coupling agents. When necessary, we use glacial acetic acid to adjust the $\mathrm{pH}$ value to make them hydrolyze sufficiently, then mix them with the solution of emulsifier, and add $\mathrm{D}_{4}$ to prepare the pre emulsion when ready to use. The influence of the solutions $\mathrm{pH}$ value is shown in Table 8 .

The droplet acceleration of pre emulsion has a great influence on the formation of polymer. The speed is too fast, which causes the uneven distribution of

Table 7. The influence of coupling agent/polyether monomer proportions dosage on the softener.

\begin{tabular}{ccccc}
\hline $\begin{array}{c}\text { W (coupling agent/ } \\
\text { polyether) }\end{array}$ & 1.25 & 2.50 & 3.00 & 5.00 \\
\hline $\begin{array}{c}\text { Centrifugal stability } \\
\text { and stratification }\end{array}$ & $\begin{array}{c}\text { Non-bleaching } \\
\text { and stratification }\end{array}$ & $\begin{array}{c}\text { Non-bleaching } \\
\text { and stratification }\end{array}$ & $\begin{array}{c}\text { Non-bleaching } \\
\text { and stratification }\end{array}$ \\
Softness (mm) & 41.7 & Translucent & Translucent & Translucent \\
Hydrophilicity & 1.02 & 27.5 & 19.2 & 19.6 \\
Whiteness (\%) & 78.6 & 1.37 & 1.59 & 4.52 \\
Tear strength $(\mathrm{N})$ & 15.4 & 78.2 & 77.5 & 76.1 \\
\hline
\end{tabular}


Table 8. The influence of the solutions $\mathrm{pH}$ value on silane coupling agent.

\begin{tabular}{ccccc}
\hline PH value of solution & $3-4$ & $5-6$ & $6-7$ & $7-8$ \\
\hline Place $1 \mathrm{~min}$ & Floc free & Floc free & Floc free & Hydrolysis \\
Place $3 \mathrm{~min}$ & Floc free & Floc free & Hydrolysis & \\
Place $5 \mathrm{~mm}$ & Floc free & Floc free & & \\
Place $10 \mathrm{~min}$ & Floc free & Floc free & & \\
Place $60 \mathrm{~min}$ & Floc free & Hydrolysis & & \\
\hline
\end{tabular}

reaction molecules, the molecular weight of polymer is too wide, and even accumulating locally. The speed is too slow, the reaction process is prolonged, the production efficiency is low, and the energy consumption is much higher and the cost is increased. After experiment, we control the pre emulsion time of 30 min.

\subsubsection{Summary of This Chapter}

In emulsion polymerization, we choose twelve alkylbenzene sulfonic acid, cationic emulsifier 1231 and 1831, and non-ionic emulsifier choose biodegradable poly-oxyethylene isomeric alcohol ether XL series. When the ratio of cationic emulsifier $1231 / 1831$ is $2.0 / 1$ and the dosage of emulsifier is $15 \%$ (for oil phase quality), the emulsifying effect is the best.

In the experiment, when the mass ratio of homemade polyether silicone oil to $\mathrm{D}_{4}$ is $1.6 / 1$, the softness of the synthetic emulsion treated fabric is the best, and the hydrophilicity is still on the rise.

In the experiment, a double amine type cinnamon coupling agent $\mathrm{KH}-602$ was selected, and its dosage was $3 \%$ of the quality of polyether silicone oil. The synthetic emulsion treated fabric had slight yellowing after high temperature baking, but its softness was excellent, and it reached the level of the marketed amino cinnamon oil softener and had good hydrophilicity.

Here we give the advantages and disadvantages of polymerization.

The polymerization speed is fast, and the molecular weight is high. With water as the medium, the viscosity is small, the cost is low, and the heat dissipation is easy; the synthetic emulsion is stable.

The process is complex, energy consumption is high and production efficiency is low. A small amount of emulsifier and other additives may be left behind.

The stability of the products synthesized by emulsion polymerization is the best. Basically, there is no layering and oil bleaching. When the fabric is treated, there will be no sticky rollers. But the handle and hydrophilicity of the fabric need further improvement.

\section{Conclusions}

1) The results show that the proportion and dosage of emulsifier, the selection and dosage of catalyst, the viscosity and hydrogen content of hydrogen-containing silicone oil, the structure and molecular weight of active polyether, the selection 
and dosage of epoxy, the selection and dosage of coupling agent and the ratio of hydrogen-containing silicone oil to active polyether have significant effects on the polymerization process and product performance.

2) The introduction of three and eight methyl ring four siloxane $\left(D_{4}\right)$ can improve the softness of softeners, but the amount of grafting is not too high. Otherwise, the hydrophilicity of the products will be affected. In the process of synthesizing hydrophilic amino silicone softeners by emulsion polymerization, the preparation of pre-emulsion must be agitated and filled. Otherwise, the coupling agent hydrolysis in Guangxi will affect the properties of softener products. Otherwise, the partial monomer reaction is incomplete and the product is easy to be stratified after being placed; the amount of coupling agent is not easy to be too large, otherwise the yellowing of the treated fabric will be serious; due to the large steric resistance of $\mathrm{D}_{4}$, the reaction will be slow after introducing $\mathrm{D} 4$, and the holding time should be prolonged to ensure the complete reaction.

3) In addition to emulsifying amino silicone oil softener products, emulsifiers also have good permeability, affecting the surface activity of polyether cinnamon oil or hydrophilic monomers. If emulsifier is not selected properly, it cannot fully emulsify cinnamon oil products, which will inevitably affect the use effect and storage stability of the emulsion. If the amount of emulsifier is not enough, it will affect the amount of graft hydrophilic groups and the emulsifying effect on the products, resulting in the decrease of the hydrophilicity, antistatic property, emulsion stability and handle of the softeners.

\section{Conflicts of Interest}

The authors declare no conflicts of interest regarding the publication of this paper.

\section{References}

[1] Lu, K., Yang, Y.M. and Huang, F. (2007) Development and Application of Silicone Softener. Hebei Textile, No. 3, 42-51.

[2] Jang, K.O. and Yeh, K. (1993) Effects of Silicone Softeners and Silane Coupling Agents on The Performance Properties of Cotton Fabrics. Textile Research, 63, 557-565. https://doi.org/10.1177/004051759306301001

[3] Dong, Y.C. (2007) Chemical and Application of Textile Auxiliaries. China Textile Press, Beijing.

[4] Xu, Q. and Jia, L.H. (2002) Dyeing Auxiliaries. Chemical Industry Press, Beijing.

[5] Zhang, X.H. and Huang, X.J. (2005) Synthesis and Application of Novel Low Yellowing Modified Amino Silicone Oil Softener. Printing Auxiliaries, 22, 7-10.

[6] Halbran, D.J. (1998) Water Soluble Ammonium Silicone Compositions and Their Use as Fiber Treatment Agents. US Patent 5707434.

[7] SWS Silicones corporation (1976) Mould Release Composition. US Patent 1453720.

[8] Sun, S.Y. and Li, J.F. (2007) Development and Application of Aminosilicic Softener. Textile Herald, No. 12, 80-86.

[9] Wang, J. and Hou, M.L. (2002) Application of Rhodorsil Hydrosoft in Textile Fa- 
bric. Dyeing Auxiliaries, 19, 39-42.

[10] Xu, Y., Cao, J. and Hao, M. (2002) Auxiliaries for Polymer Synthesis. Chemical Industry Press, Beijing.

[11] Su, Y.G. (2007) Synthesis of High Viscosity Amino Silicone Oil. Silicone Material, 21, 33-35.

[12] Sawada, K. and Ueda, M. (2003) Adsorption Behavior of Direct Dye on Cotton in Non-Aqueousm Media. Dyes and Pigment, 58, 37-40. https://doi.org/10.1016/S0143-7208(03)00027-5

[13] Sawada, K. and Ueda, M. (2001) Enzyme Processing of Textiles in Reverse Micellar Solution. Journal of Biotechnology, 89, 263-269. https://doi.org/10.1016/S0168-1656(01)00310-8

[14] Luo, J.T. (2000) Dyeing Auxiliaries and Their Application. China Textile Press, Beijing.

[15] Su, H., Yang, X.Y. and Qu, J. (2002) Synthesis and Application of Linear Polymerized Amino Silicone Oil. Dyeing Auxiliaries, 19, 46-47.

[16] Li, Y., Liu, J.X. and Wan, R. (2008) Preparation and Application of Hydrophilic Amino Silicone Oil. Dyeing Auxiliaries, 25, 19-21.

[17] Luo, Y.J. and Gui, H.X. (2002) Silicone Resin and Its Application. Chemical Industry Press, Beijing. 